\title{
CARACTERÍSTICAS DE LAS COSTRAS FÍSICAS Y BIOLÓGICAS DEL SUELO CON MAYOR INFLUENCIA SOBRE LA INFILTRACIÓN Y LA EROSIÓN EN ECOSISTEMAS SEMIÁRIDOS
}

S. Chamizo ${ }^{1}$, E. Rodríguez-Caballero ${ }^{2}$, I. Miralles-Mellado ${ }^{3}$, A. AfanA ${ }^{1}$, R. LÁzaro ${ }^{1}$, F. Domingo ${ }^{1,5}$, A. CAlvo-Cases ${ }^{4}$, A. SOle-Benet ${ }^{1} \&$ Y. CANTÓN $^{2}$

1 Estación Experimental de Zonas Áridas, CSIC, Almería, España 2 Departamento de Edafología y Química Agrícola, Universidad de Almería, España 3 Departamento de Geografía Física de la Universidad Católica de Louvain-la-Neuve, Bélgica

4 Departamento de Geografía, Universidad de Valencia, España 5 Departamento de Biología Vegetal y Ecología, Escuela Politécnica Superior, Universidad de Almería, España.

Email.: schamizo@eeza.csic.es

ABSTRACT.- Physical soil crusts and biological soil crusts occupy a wide extension in arid and semiarid areas all over the world. In these regions, soil crusting has a very strong influence on local hydrologic regimes and erosion. The main purposes of this paper are: 1) to examine how crust characteristics influence infiltration and erosion and 2) to identify the most influential crust characteristics on runoff and erosion processes. Two semiarid areas in the province of Almeria were chosen and the most representative physical and biological soil crusts were identified at both sites. For each crust type, physical and chemical characteristics of the crust and the soil underneath the crust were analysed, as well as other crust properties like roughness, hydrophobicity, resistance to penetration and cover. To analyse the influence of the crust on infiltration and erosion, rainfall simulations were conducted on plots with the intact crust, and in order to examine the influence of the soil underlying the crust, rainfall simulations were conducted on plots after removing the crust. The hydrological response of the studied encrusted areas is affected, not only by the characteristics of the crust itself, but also by the characteristics of the soil in where the crust is developed and specially by the slope gradient that appears like a highly predictive variable for infiltration and erosion. Among the properties of the soil material below crusts, it is remarkable the influence of soil texture, organic carbon content and electrical conductivity, and among the properties of the crust, cover and roughness are the most predictive variables explaining the differences in infiltration and erosion between sites. 
Keywords: Soil crusting; Biological soil crust; Runoff; Erosion; Semiarid.

\begin{abstract}
RESUMEN.- Las costras físicas (CFS) y biológicas (CBS) del suelo ocupan una gran extensión en zonas áridas y semiáridas de todo el mundo. En estos medios, el encostramiento del suelo tiene una gran influencia sobre los procesos hidrológicos y erosivos. Los objetivos que se persiguen en este trabajo son: analizar las características de las costras que influyen en la infiltración y en la erosión e identificar cuáles de estas características tiene una mayor influencia sobre estos procesos. En dos áreas semiáridas representativas en la provincia de Almería se identificaron los principales tipos de costras físicas y biológicas. Para cada tipo de costra, se analizaron las características físicas y quimicas de la propia costra y del material subyacente, así como su rugosidad, hidrofobia, resistencia a la penetración y cobertura de diferentes comunidades de organismos. Para analizar la influencia de las costras sobre la infiltración y la erosión, se llevaron a cabo simulaciones de lluvia en parcelas con la costra intacta y para examinar los efectos de las características del material subyacente a la costra, los experimentos de simulación de lluvia se realizaron tras retirar la costra. La respuesta hidrológica de las áreas encostradas estudiadas se ve afectada no solo por las características de la costra, sino también por las características del suelo sobre el que se desarrollan estas costras y especialmente por la pendiente que aparece como una variable altamente predictiva para la infiltración y la erosión. Entre las características del material subyacente, resalta la influencia de la textura, el contenido en carbono orgánico y la conductividad eléctrica, y entre las características de la costra, la cobertura y rugosidad son las variables más predictivas que explican las diferencias en las tasas de infiltración y erosión entre ambos sitios.
\end{abstract}

Palabras clave: Costra física; costra biológica; escorrentía; erosión; semiárido.

\title{
1. Introducción
}

Las condiciones de la superficie del suelo como el encostramiento, la rugosidad, la pedregosidad y/o la cobertura vegetal, tienen una gran influencia en los regímenes hidrológicos locales, ya que controlan los procesos de infiltración, generación de escorrentía y erosión (AUZET et al., 2005; LE BISSONNAIS et al., 2005). En los ecosistemas áridos y semiáridos, donde la cobertura vegetal es escasa, aparecen numerosos claros desprovistos de vegetación, por lo que otros componentes de la superficie del suelo, como la pedregosidad o las costras, adquieren un papel fundamental en el control de la escorrentía, la distribución del agua, la velocidad de flujo y la erosión. En estos sistemas, el suelo de las zonas desprovistas de vegetación se encuentra a menudo cubierto por costras tanto físicas (CFSs) como biológicas (CBSs), las cuales son un consorcio de hongos, algas, cianobacterias, musgos, líquenes y otros organismos. Las CBSs son muy comunes en muchos 
ecosistemas dentro de un amplio rango bioclimático, que abarca desde los desiertos a las regiones polares (BELNAP et al., 2001) y pueden representar hasta el 70\% de la cobertura en estos medios. Además, las CBSs se consideran los primeros colonizadores en ecosistemas que han sufrido alteraciones de gran magnitud (VESTE, 2005).

Las costras biológicas del suelo tienen un papel fundamental en el control de los procesos hidrológicos (BELNAP, 2006; YAIR, 2008) y de la erosión. En las últimas décadas se han publicado varios trabajos que analizan el efecto de las CFSs y las CBSs sobre los procesos de infiltración y erosión a escala de parcela (ALEXANDER \& CALVO-CASES, 1990; ELDRIDGE \& GREENE, 1994; KIDRON \& YAIR, 2001; FOX et al., 1998; ELDRIDGE et al., 2000; CANTON et al., 2001 \& 2002; NDIAYE et al., 2005; LE BISSONNAIS et al., 2005; BELNAP, 2006), en los que se han identificado de forma repetida como principales responsables de la respuesta hidrológica y erosiva observada algunas características de las costras. Los efectos de las costras sobre los procesos de erosión e infiltración están fuertemente influenciados por: i) las condiciones locales (WARREN, 2003), las características tanto físicas (textura, estructura, densidad aparente, estabilidad de los agregados, etc.) como químicas (carbono orgánico total, porcentaje de sodio intercambiable), de los primeros centímetros de suelo; ii) la proporción de suelo ocupada por las costras, composición de la costra, biomasa de cianobacterias (SOLÉ-BENET et al., 1997; LÁZARO et al., 2008); iii) la hidrofobia (KIDRON et al., 1999) de las costras y iv) la rugosidad superficial (BELNAP, 2006). Todos estos parámetros interactúan de una manera compleja haciendo que sea difícil predecir la respuesta hidrológica de un tipo específico de CBS y más aún de mosaicos con distintas proporciones de CBSs y CFSs que es como aparecen habitualmente en muchos ecosistemas.

El objetivo de este trabajo es analizar la relación de diferentes características de las costras y del material subyacente con la infiltración y la erosión e identificar cuáles de estas características tiene una mayor influencia sobre estos procesos.

\section{Material y métodos}

\section{1. Áreas de estudio}

Se seleccionaron dos ecosistemas semiáridos mediterráneos en la provincia de Almería (España) donde están bien representados los procesos de encostramiento: 
A) El Cautivo: Zona de badlands localizada en el desierto de Tabernas, en la depresión Neógeno-Cuaternaria que limita al norte con Sierra de Filabres y al sur con Sierra de Alhamilla. La cuenca se rellenó principalmente con sedimentos marinos del Neógeno (KLEVERLAAN, 1989), y con material de origen continental. Los badlands se han ido formado durante el Cuaternario sobre margas yesíferas escasamente estratificadas del Tortoniense (CANTÓN et al., 2001 \& 2002). El clima de la zona es termomediterráneo semiárido (LÁZARO \& REY, 1990) con veranos largos y secos, una temperatura media anual de $17.9^{\circ} \mathrm{C}$ y una precipitación media anual de $235 \mathrm{~mm}$, que se concentra principalmente en invierno. La evapotranspiración potencial anual es de unos $1500 \mathrm{~mm}$, por lo que existe un déficit hídrico anual importante (CANTÓN et al., 2003). El desierto de Tabernas presenta una gran heterogeneidad en la cobertura de la superficie del suelo, y se presenta como un mosaico de teselas dominadas por plantas perennes dispersas, plantas anuales y costras físicas y biológicas, que llegan a cubrir más del $80 \%$ de la superficie del suelo (CANTÓN et al., 2004). El paisaje, caracterizado por su accidentada topografía, está formado por valles asimétricos de dirección NW$\mathrm{SE}$, con laderas orientadas al SW con poca pendiente y laderas orientadas al NE con pendientes elevadas. En las partes más altas de las primeras se desarrollan suelos incipientes (Regosoles Endolépticos) cubiertos principalmente por costras de líquenes y algunas plantas anuales y perennes. En las partes bajas de las laderas, donde las pendientes son más suaves, los tipos de suelos dominantes son los Calcisoles Háplicos, cubiertos principalmente por plantas anuales y arbustos enanos o herbáceas perennes dispersas y costras físicas y biológicas en los claros. Las laderas orientadas al SW alcanzan hasta $70^{\circ}$ de pendiente y presentan suelos muy poco desarrollados (Regosoles Epilépticos) donde dominan la CFSs y localmente aparecen manchas de líquenes o plantas anuales (CANTÓN et al., 2003 \& 2004).

Las costras más representativas identificadas en la zona fueron: 1) Costra estructural sobre margas (E); 2) Costra deposicional con un grado incipiente de colonización por cianobacterias (CI); 3) Costra biológica dominada por cianobacterias (C), y 4) Costra biológica dominada por líquenes (L). La primera es una costra física y las demás son costras biológicas, ordenadas de menos a más evolucionadas.

B) Amoladeras, localizada en el Parque Natural Cabo de Gata-Nijar. Se trata de una zona llana sobre un encostramiento calcáreo de la parte distal del abanico aluvial de Sierra Alhamilla. Los suelos son delgados (aproximadamente $10 \mathrm{~cm}$ de media hasta un máximo de $30 \mathrm{~cm}$ ) y muy pedregosos. Los tipos de suelos dominantes son Leptosoles Eútricos y Réndsicos; están cubiertos principalmente por matorral disperso de pequeño 
porte, dominado a menudo por Stipa tenacissima, y presentan costras biológicas ocupando los espacios libres entre las plantas. El clima es mediterráneo semiárido, con una precipitación media anual de unos $200 \mathrm{~mm}$ y una temperatura media anual de $18{ }^{\circ} \mathrm{C}$, caracterizado por veranos muy secos y prolongados y por las escasas y aleatorias precipitaciones.

Las costras más representativas identificadas en Amoladeras fueron costras biológicas dominadas por cianobacterias (C), costras biológicas con predominancia de líquenes (L) y costras biológicas dominadas por musgos (M).

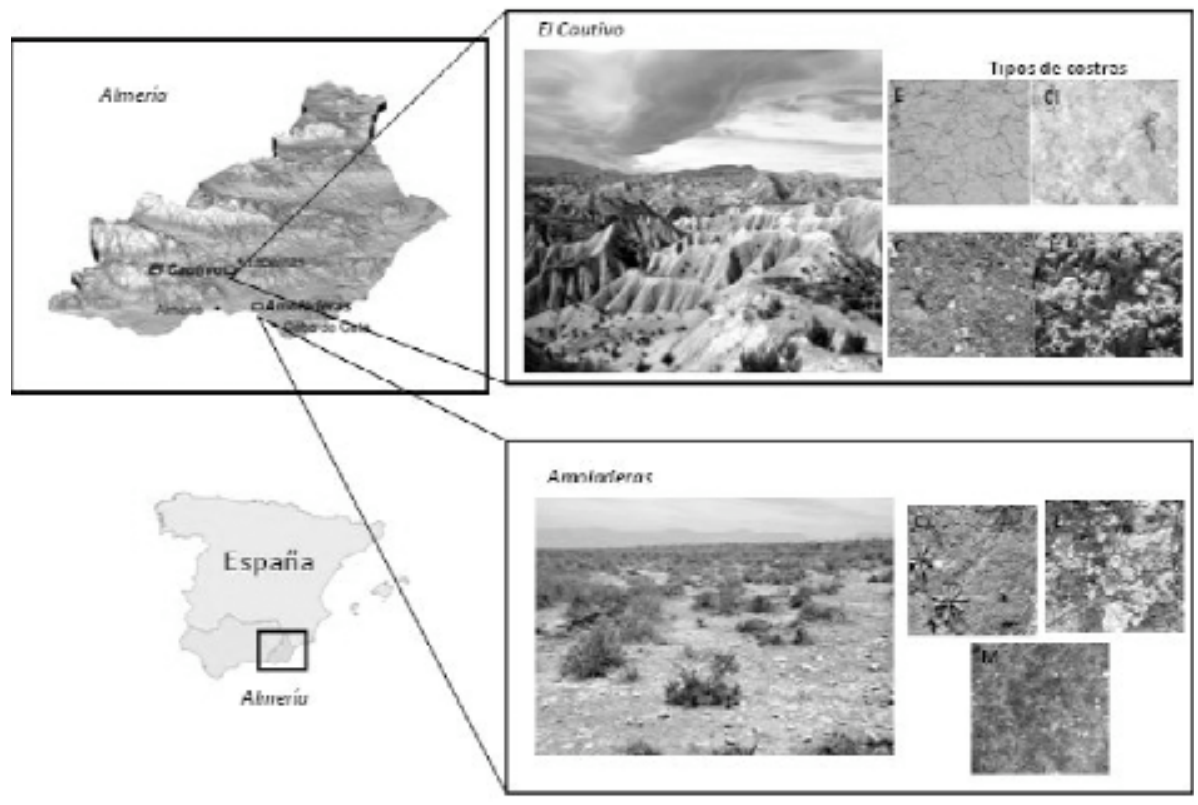

Figura 1. Mapa de localización e imagen de las áreas de estudio y principales tipos de costras identificados en cada área.

Figure 1. Location map and pictures of the study areas and of the main crust types identified at each site.

\subsection{Análisis de las características de las costras}

\subsubsection{Material subyacente}

Para analizar las propiedades fisicoquímicas del material subyacente a la costra biológica, se tomaron cuatro muestras del primer centímetro de suelo 
bajo los diferentes tipos de costras, en ambas áreas de estudio. En cada una de las muestras se determinó el contenido en carbono orgánico total (COT) mediante oxidación con dicromato potásico (método de WALKLEY \& BLACK (1934), modificado por BARAHONA et al., 2005). El porcentaje de las distintas fracciones granulométricas se determinó empleando el método de la pipeta de Robinson (GEE \& BAUDER, 1986) y el contenido de carbonato total con el calcímetro de Bernard (LOEPPERT \& SUAREZ, 1996). La capacidad de intercambio catiónico (CIC) y las bases de cambio se determinaron tras la extracción con soluciones de acetato amónico (SUMNER \& MILLER, 1996). Los cationes $\mathrm{Na}^{+}, \mathrm{K}^{+}, \mathrm{Ca}^{2+}$ y $\mathrm{Mg}^{2}$ fueron medidos por espectrofotometría de absorción atómica. La capacidad de retención de agua del suelo (CRA) se determinó a $-33 \mathrm{kPa}$ y $-1500 \mathrm{kPa}$ mediante el método de la membrana de RICHARDS (1945) y el pH se midió en una suspensión suelo-agua en proporción de peso 1:1.

\subsubsection{Costras}

Sobre cada uno de los tipos de costras identificados se delimitaron cuatro parcelas circulares de $0.25 \mathrm{~m}^{2}$ y en cada una de ellas se midió la pendiente con un clinómetro y se estimó la cobertura de costra física, cianobacterias, líquenes, musgo, piedras y anuales presentes usando una malla de $0.5 \times 0.5$ metros, dividida en cuadriculas de 0.5 decímetros de lado.

Se midió la hidrofobia de la costra en campo mediante el test de penetración de gotas (WDPT, Water Drop Penetration Time), que consiste en cuantificar el tiempo que tarda en penetrar una gota de agua en una muestra de suelo y así establecer el grado de repelencia al agua que presenta un suelo. En cada parcela se identificaron los principales tipos de coberturas y su superficie y se hicieron siete repeticiones del test de la gota en cada una de ellas. Se hizo una media ponderada del WDPT con la superficie y finalmente se calculó el WDPT en cada costra como el valor medio de las cuatro parcelas.

La rugosidad de la costra se determinó mediante el método de la cadena, que consiste en medir la diferencia entre la longitud total de una cadena y la longitud horizontal entre el comienzo y el final de la cadena cuando se coloca en línea sobre el suelo. Se utilizó una cadena con una longitud de $50 \mathrm{~cm}$ y con un tamaño de eslabón de $3 \mathrm{~mm}$, y en cada parcela se realizaron tres transectos de rugosidad en la dirección de la pendiente y otras tres medidas de rugosidad transversales (perpendiculares a las anteriores). Se calculó el valor medio de la rugosidad en cada una de las direcciones en cada tipo de costra.

En sitios adyacentes a las parcelas, sobre los mismos tipos morfológicos de costra, se realizaron 15 medidas de resistencia de la costra, usando un 
penetrómetro de bolsillo Eijelkamp con un cono de 6,4 $\mathrm{mm}$ de diámetro. Y además se tomaron muestras de cada uno de los tipos de costra identificados para analizar posteriormente el contenido en carbono orgánico total, nitrógeno total y capacidad de retención de agua de la costra a -33 kPa y -1500 $\mathrm{kPa}$.

\subsubsection{Comportamiento hidrológico y erosivo de las costras}

En las parcelas descritas anteriormente, se colocaron aros circulares de $0.25 \mathrm{~m}^{2}$ que disponían de un aforo. En cada parcela se realizaron dos experimentos de simulación de lluvia sucesivos, ambos de 30 minutos de duración y con una intensidad de unos $50 \mathrm{~mm} \mathrm{~h}^{-1}$, el primero sobre suelo seco y el segundo, 30 minutos después, sobre el suelo húmedo. Esta intensidad de lluvia tiene un periodo de retorno de cinco años (LÁZARO et al., 2001). Se midió el inicio de la escorrentía de cada parcela al comienzo de cada evento y, una vez iniciada la escorrentía, se registró su volumen cada minuto al principio y cada 2 ó 3 minutos tras los primeros 15 minutos y se tomaron muestras de agua a lo largo del experimento con el objetivo de analizar posteriormente los sedimentos en el laboratorio. Se utilizaron simuladores basados en el diseñado por CALVO-CASES et al. (1988), que consiste en una boquilla de aspersión que llueve sobre un área circular de $1 \mathrm{~m}^{2}$, pero en nuestro caso nos centramos sólo en el círculo central de $0,25 \mathrm{~m}^{2}$ donde la lluvia es más uniforme. Para la realización del experimento, se rodearon los simuladores con plásticos para evitar la alteración de las trayectorias de las gotas por efecto del viento. El agua era conducida al simulador mediante una bomba de presión y la presión del agua que entraba a la boquilla del simulador era regulada mediante manómetros.

Además para analizar los efectos de las características del material subyacente sobre la infiltración y la erosión, se realizaron experimentos de simulación de lluvia con las mismas características que los descritos previamente, en parcelas circulares de $0.25 \mathrm{~m}^{2}$ donde se eliminó la costra.

\subsubsection{Análisis estadísticos}

Se han aplicado modelos de regresión simple para examinar el efecto de las diferentes variables, y de regresión múltiple paso a paso para la selección de las variables más determinantes en la predicción de la erosión e infiltración mediante el programa Statistica (StatSoft Inc., 2004). Debido a que los modelos de regresión múltiple por pasos sobreestiman la fuerza de las 
relaciones (LARK et al., 2007; VAN SCHAIK, 2009), especialmente cuando hay muchas variables en relación con el número de muestras, se han restringido los modelos de regresión múltiple a variables independientes significativas seleccionadas del material subyacente y propiedades de las costras, tanto para la erosión como para la infiltración. Finalmente, los resultados de análisis de correlación entre variables y los resultados de las regresiones múltiples paso a paso permitieron escoger el mejor modelo.

\section{Resultados}

\subsection{Influencia de las características del material subyacente y de la pendiente}

La tabla 1 recoge los valores medios, así como la desviación estándar, de la pendiente y de las características analizadas del material subyacente, junto con el resultado de la regresión de dichas variables con las tasas de infiltración y erosión, tras eliminar la costra superficial del suelo. La pendiente, el COT, la textura del suelo, el contenido en carbonatos y la conductividad resultan las variables más predictivas para la erosión y la infiltración de las parcelas una vez eliminada la costra.

El análisis de regresión múltiple con la tasa de infiltración como variable dependiente, muestra una $\mathrm{r}^{2}$ de 0.54 y da como variables independientes más significativas para la predicción de la tasa de infiltración, la pendiente, el carbono orgánico, el contenido en limos, los carbonatos y la conductividad eléctrica. Mientras que la $\mathrm{r}^{2}$ obtenida para la erosión como variable dependiente es de $65 \%$ y la pendiente, el carbono orgánico, las arcillas y el contenido en carbonatos aparecen como las variables independientes más significativas.

\subsection{Influencia de las características de las costras}

\subsubsection{Cobertura y composición de las costras}

La tabla 2 muestra la tasa de infiltración tras 1 hora de lluvia simulada en distintas parcelas experimentales, con distintos porcentajes de cobertura de cianobacterias.

Las mayores tasas de escorrentía aparecen en las parcelas con costra física; la infiltración aumenta cuando lo hace la superficie cubierta por 


\section{CARACTERÍSTICAS DE LAS COSTRAS FÍSICAS Y BIOLÓGICAS DEL SUELO CON MAYOR...}

cianobacterias. Si además atendemos a la producción de sedimentos (Figura 2), ésta es mucho mayor en la costra física y disminuye al aumentar la cobertura de cianobacterias.

Tabla 1. Media y desviación estándar de la pendiente y de las características analizadas del material subyacente para las dos áreas, así como el resultado de la regresión de dichas variables con las tasas de infiltración y erosión de estos suelos tras eliminar la costra. p: p-valor; CC: coeficiente de correlación; ns: no significativo cuando p es mayor de 0.05 .

Table 1. Mean and standard deviation of the slope gradient and the analysed characteristics of the underlying material for both sites, as well as the regression model results of each characteristic with the infiltration and erosion rates of these soils after the crust removal. $p$ : $p$-value; CC: correlation coefficient; ns: non-significant when $p$ is higher than 0.05 .

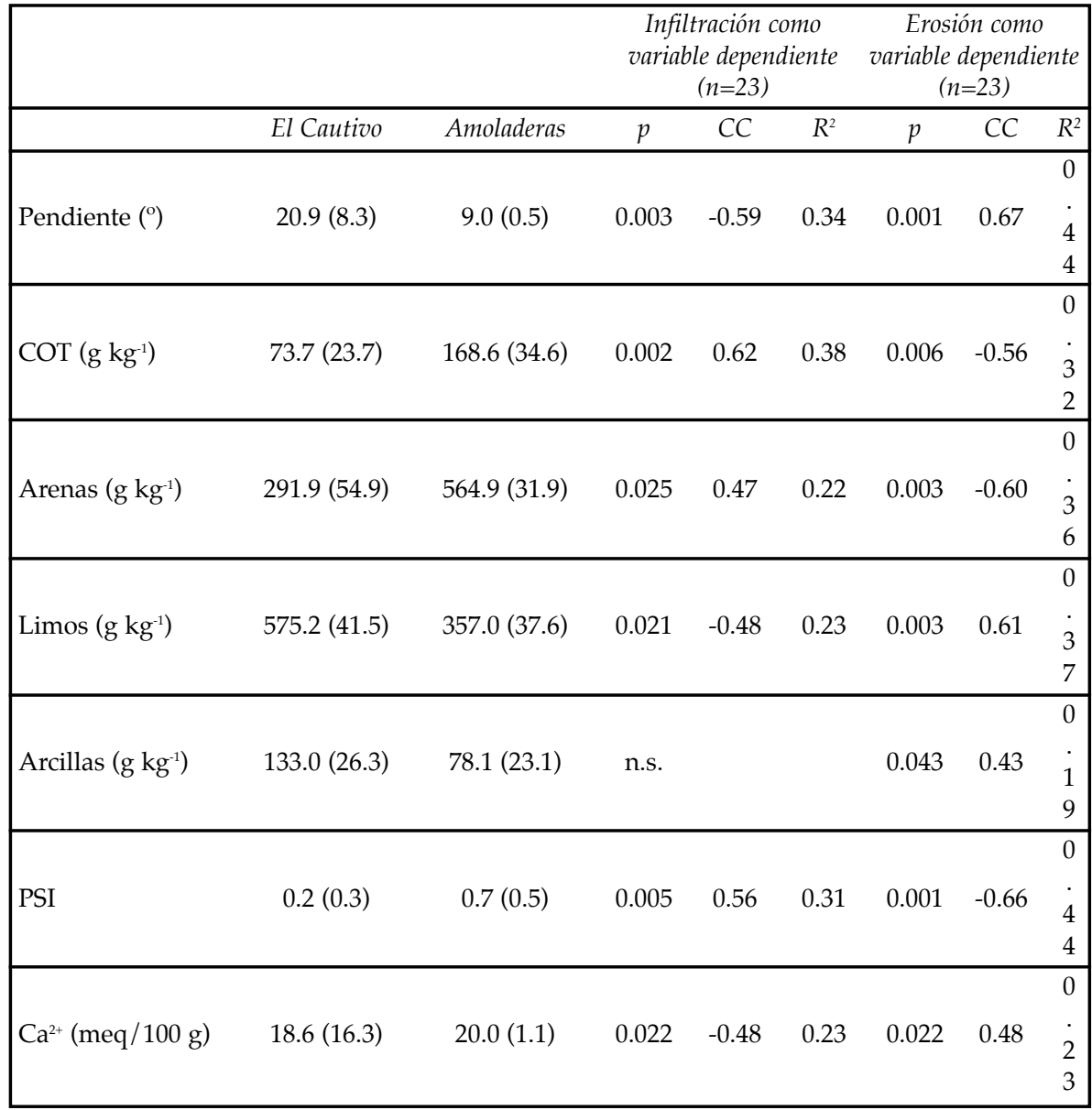


S. CHAMIZO, E. RODRÍGUEZ, I. MIRALLES, A. AFANA, R. LÁZARO, F. DOMINGO, A. CALVO, A. SOLE \& Y. CANTÓN

\begin{tabular}{|c|c|c|c|c|c|c|c|c|}
\hline & \multirow[b]{2}{*}{ El Cautivo } & \multirow[b]{2}{*}{ Amoladeras } & \multicolumn{3}{|c|}{$\begin{array}{c}\text { Infiltración como } \\
\text { variable dependiente } \\
\qquad(n=23)\end{array}$} & \multicolumn{3}{|c|}{$\begin{array}{c}\text { Erosión como } \\
\text { variable dependiente } \\
(n=23)\end{array}$} \\
\hline & & & $p$ & $C C$ & $R^{2}$ & $p$ & $C C$ & $R^{2}$ \\
\hline $\mathrm{Mg}^{2+}(\mathrm{meq} / 100 \mathrm{~g})$ & $0.6(0.0)$ & $0.6(0.0)$ & n.s. & & & n.s. & & \\
\hline $\mathrm{K}^{+}(\mathrm{meq} / 100 \mathrm{~g})$ & $0.7(0.1)$ & $0.9(0.1)$ & n.s. & & & 0.027 & -0.47 & $\begin{array}{l}0 \\
2 \\
2 \\
2\end{array}$ \\
\hline $\mathrm{Na}^{+}(\mathrm{meq} / 100 \mathrm{~g})$ & $0.4(0.1)$ & $0.5(0.1)$ & n.s. & & & 0.044 & -0.43 & $\begin{array}{l}0 \\
. \\
1 \\
8\end{array}$ \\
\hline Carbonatos $\left(\mathrm{g} \mathrm{kg}^{-1}\right)$ & $244.4(30.6)$ & $130.9(15.0)$ & n.s. & & & 0.056 & 0.41 & $\begin{array}{l}0 \\
. \\
1 \\
7\end{array}$ \\
\hline $\mathrm{pH}$ & $7.7(0.3)$ & $7.8(0.3)$ & n.s. & & & n.s. & & \\
\hline $\begin{array}{l}\text { Conductividad } \\
\left(\mu S \mathrm{~m}^{-1}\right)\end{array}$ & $841.3(784.3)$ & $174.9(33.2)$ & 0.002 & -0.60 & 0.36 & n.s. & & \\
\hline $\begin{array}{l}\text { CRA -33 kPa } \\
\left(\mathrm{cm}^{3} \mathrm{~cm}^{-3}\right)\end{array}$ & $28.3(3.7)$ & $24.3(1.4)$ & n.s. & & & n.s. & & \\
\hline $\begin{array}{l}\text { CRA -1500 kPa } \\
\left(\mathrm{cm}^{3} \mathrm{~cm}^{-3}\right)\end{array}$ & $8.8(1.6)$ & $9.0(0.9)$ & n.s. & & & n.s. & & \\
\hline $\begin{array}{l}\text { Tasa de infiltración } \\
\left(\mathrm{mmh}^{-1}\right)\end{array}$ & $22.4(9.5)$ & $30.5(6.6)$ & & & & & & \\
\hline $\begin{array}{l}\text { Tasa de erosión } \\
\left(\mathrm{g} \mathrm{m}^{-2}\right)\end{array}$ & $350.7(258.5)$ & $40.1(45.6)$ & & & & & & \\
\hline
\end{tabular}

Tabla 2. Cobertura de la costra y tasa de infiltración bajo condiciones de lluvia simulada en El Cautivo.

Table 2. Crust cover and infiltration rates under simulated rainfall in El Cautivo.

\begin{tabular}{|ccc|}
\hline $\begin{array}{c}\text { Cobertura de costra física } \\
(\%)\end{array}$ & $\begin{array}{c}\text { Cobertura de cianobacterias } \\
(\%)\end{array}$ & $\begin{array}{c}\text { Tasa de infiltración } \\
\left(\mathrm{mm} \mathrm{h}^{-1}\right)\end{array}$ \\
\hline 100 & 0 & 9.13 \\
80 & 15 & 18.72 \\
53 & 40 & 22.56 \\
10 & 86 & 32.65 \\
\hline
\end{tabular}




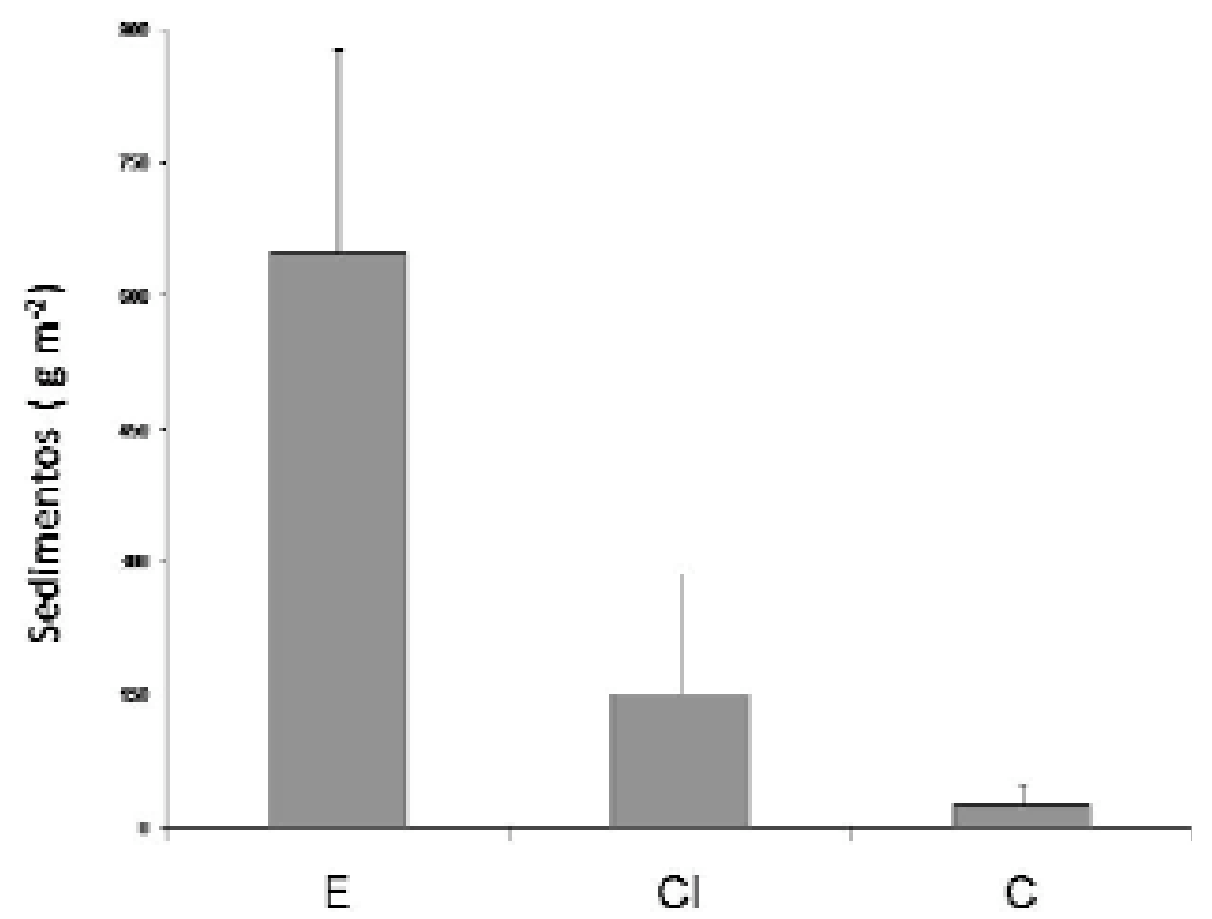

Figura 2. Diferentes tipos de costra en El Cautivo (E: costra física, CI: Costra de cianobacterias incipiente, C: costra dominada por cianobacterias) y tasa media de erosión obtenida con simulación de lluvia.

Figure 2: Different crust types in El Cautivo (physical crust, cyanobacteria-incipient crust, cyanobacteriadominated crust) and total average erosion rates obtained with simulated rainfall.

La composición biológica de las CBSs también juega un papel fundamental sobre la respuesta hidrológica y erosiva de la costra. En la figura 3 se representan la media y el percentil 75 de las tasas de infiltración y erosión en los tres tipos de costra biológica identificados en Amoladeras, y se observa que las diferencias son significativas entre los tres tipos de costra tanto en la infiltración como en la producción de sedimentos. Los valores más altos de infiltración y los más bajos de erosión corresponden a las costras dominadas por musgo. 


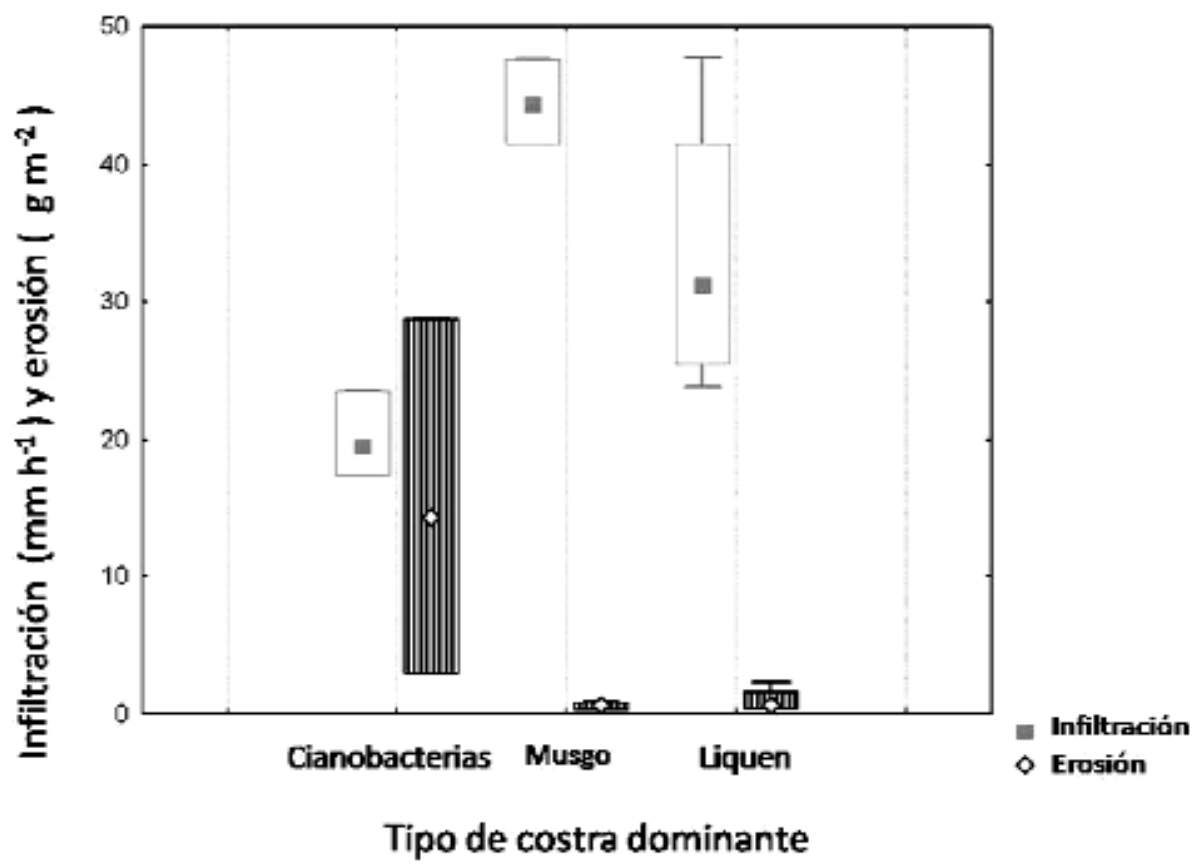

Figura 3. Media y percentil 75 de las tasas de infiltración y erosión en los tres tipos de costras biológicas en Amoladeras.

Figure 3. Mean and 75 percentile of infiltration and erosion rates in the three types of biological soil crusts in Amoladeras.

\subsubsection{Hidrofobia}

Aunque en general puede decirse que los tipos de costra identificadas en nuestras áreas de estudio no son hidrofóbicas según la clasificación propuesta por DEBANO (1981), encontramos una correlación negativa entre el WDPT y el umbral de precipitación $(\mathrm{mm})$ para iniciar la escorrentía, así como entre el WDPT y la tasa de infiltración (Figura 4). Además, el tiempo necesario para la penetración de la gota de agua en la costra es mayor y la infiltración y la cantidad de lluvia necesaria para el inicio de la escorrentía son menores en el caso de las costras de líquenes que en otras costras menos desarrolladas y más tempranas en la sucesión vegetal como las costras de cianobacterias 


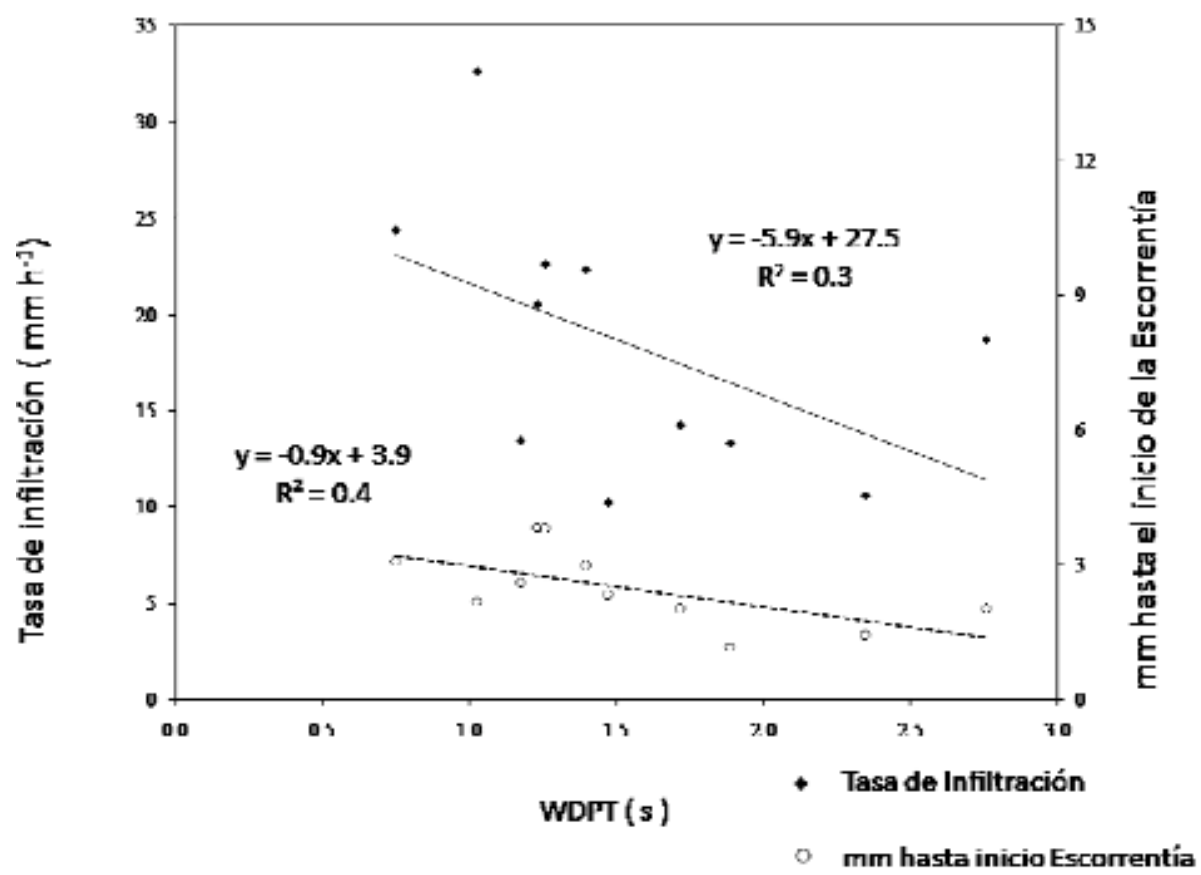

Figura 4. Tiempo de penetración de la gota en las costras de El Cautivo frente a las tasas de infiltración y la cantidad de lluvia necesaria $(\mathrm{mm})$ para el inicio de la escorrentía.

Figure 4. Water drop penetration time in the crusts from El Cautivo vs. infiltration rates and amount of rain $(\mathrm{mm})$ to generate runoff.

\subsubsection{Resistencia a la penetración de las costras}

En la tabla 3 se presentan los resultados de la resistencia a la penetración de los distintos tipos de costra. Al aumentar el grado de desarrollo de la costra, la resistencia disminuye y también lo hace la erosión. La relación de la resistencia con la escorrentía no es tan clara, de forma que a medida que disminuye la resistencia, disminuye la escorrentía, a excepción de las costras dominadas por líquenes. No se observa una relación clara entre la resistencia a la penetración de la costra y la lluvia necesaria para el inicio de la escorrentía. 
Tabla 3. Media y desviación estándar de la resistencia mecánica, infiltración y erosión tras 1 hora de lluvia simulada y $\mathrm{mm}$ hasta inicio de la escorrentía en los diferentes tipos de costras morfológicas en El Cautivo. La rugosidad media (vertical y horizontal) se presenta como un índice adimensional (longitud medida del perfil en $\mathrm{cm} /$ longitud proyectada en $\mathrm{cm}$ ).

Table 3. Mean and standard deviation of penetration resistance, infiltration and erosion rates after 1 hour of simulated rainfall and rain to runoff $(\mathrm{mm})$ on the different morphological types of El Cautivo crusts. Mean roughness (vertical and horizontal) is expressed by a dimensionless index (measured profile length in $\mathrm{cm} /$ projected length in $\mathrm{cm}$ ).

\begin{tabular}{|ccccccccc|}
\hline $\begin{array}{c}\text { Tipo } \\
\text { de } \\
\text { costra }\end{array}$ & $\begin{array}{c}\text { Resistencia } \\
\text { mecánica } \\
\left(\mathrm{kg} \mathrm{cm}^{-2}\right)\end{array}$ & $\begin{array}{c}\text { Rugosidad } \\
\text { vertical }\end{array}$ & $\begin{array}{c}\text { Rugosidad } \\
\text { horizontal }\end{array}$ & $\begin{array}{c}\text { Tasa de } \\
\text { infiltración } \\
\left(\mathrm{mm} \mathrm{h}^{-1}\right)\end{array}$ & $\begin{array}{c}\text { mm hasta } \\
\text { escorrentía } \\
(\text { suelo seco) })\end{array}$ & $\begin{array}{c}\text { mm hasta } \\
\text { escorrentía } \\
(\text { suelo húmedo })\end{array}$ & $\begin{array}{c}\text { Erosión por } \\
\text { salpicadura } \\
\left(\mathrm{g} \mathrm{m} \mathrm{m}^{-2} \text { año }\right)\end{array}$ & $\begin{array}{c}\text { Erosión } \\
\text { laminar } \\
\left(\mathrm{g} \mathrm{m} \mathrm{m}^{-2}\right)\end{array}$ \\
\hline $\mathrm{E}$ & $1.85 \pm 0.35$ & 1.05 & 1.06 & $13.27 \pm 0.8$ & $2.08 \pm 0.34$ & $0.71 \pm 0.68$ & 2191.4 & $647.8 \pm 230.5$ \\
$\mathrm{C}$ & $2.65 \pm 0.41$ & 1.09 & 1.11 & $23.19 \pm 7.9$ & $2.70 \pm 0.40$ & $1.72 \pm 0.58$ & 1029.28 & $26.0 \pm 23.4$ \\
$\mathrm{~L}$ & $1.32 \pm 0.35$ & 1.18 & 1.22 & $13.67 \pm 4.81$ & $2.18 \pm 1.21$ & $0.90 \pm 0.29$ & 236.75 & $10.7 \pm 3.1$ \\
\hline
\end{tabular}

\subsubsection{Rugosidad de la costra}

En la tabla 3 observamos los valores de rugosidad de los diferentes tipos de costras en El Cautivo. Los valores más bajos de rugosidad se dan en las CFSs, aumentando la rugosidad progresivamente con el grado de desarrollo de la costra, hasta alcanzar los valores más elevados en los líquenes (la costra más desarrollada en Tabernas). Cabría esperar una relación directa entre la rugosidad superficial y la lluvia necesaria para el inicio de la escorrentía, sin embargo, esta relación sólo es apreciable cuando se comparan las CBSs frente a las CFSs. Si comparamos las costras biológicas entre sí, observamos que los líquenes, con una rugosidad superficial mayor, requieren menor cantidad de lluvia para iniciar la escorrentía y tienen una menor tasa de infiltración que las cianobacterias, las cuales presentan una rugosidad superficial menor. La relación de la rugosidad con otros parámetros como la erosión laminar y la erosión por salpicadura es negativa.

\subsection{Valor predictivo de las características de la costra}

Para identificar la combinación de las características tanto de la costra superficial como del material subyacente que prediga de una manera más certera las tasas de infiltración y escorrentía en los ecosistemas de estudio, se realizaron dos análisis de regresión múltiple, uno incluyendo ambos ecosistemas y otro sólo en El Cautivo, por haber una mayor disponibilidad de datos y de parcelas. En las tablas 4 y 5, se observa el resultado del análisis de regresión simple para cada parámetro en ambos ecosistemas y en El Cautivo, 
respectivamente. Sólo se muestran las regresiones que fueron significativas a un nivel de confianza del 95\%. Cuando consideramos ambos ecosistemas (Tabla 4), sólo se ve una regresión significativa con la pendiente y algunas características del material subyacente como el COT, la granulometría, el contenido en carbonatos y la conductividad eléctrica. Por lo tanto, la influencia sobre la infiltración de las características mencionadas del material subyacente se mantiene aún cuando la superficie se encuentra cubierta por costras. Algunas características de las propias costras muestran también relaciones significativas con la infiltración. La cobertura de la costra, su rugosidad, así como algunas propiedades fisicoquímicas de la costra como el COT y la CRA a - $1500 \mathrm{KPa}$ muestran una relación significativa con la infiltración. El número de variables significativas relacionadas con la erosión es menor.

Tabla 4. Variables significativas tras el análisis de regresión simple de las características de la costra y el material subyacente con las tasas de infiltración y erosión, considerando ambos ecosistemas. p: p-valor; CC: coeficiente de correlación; ns: no significativo cuando p es mayor de 0.05 .

Table 4. Significant variables after conducting a simple regression model of the characteristics of the crust and the underlying material with infiltration and erosion rates, considering both ecosystems. p: p-value; CC: correlation coefficient; ns: non-significant when $p$ is higher than 0.05 .

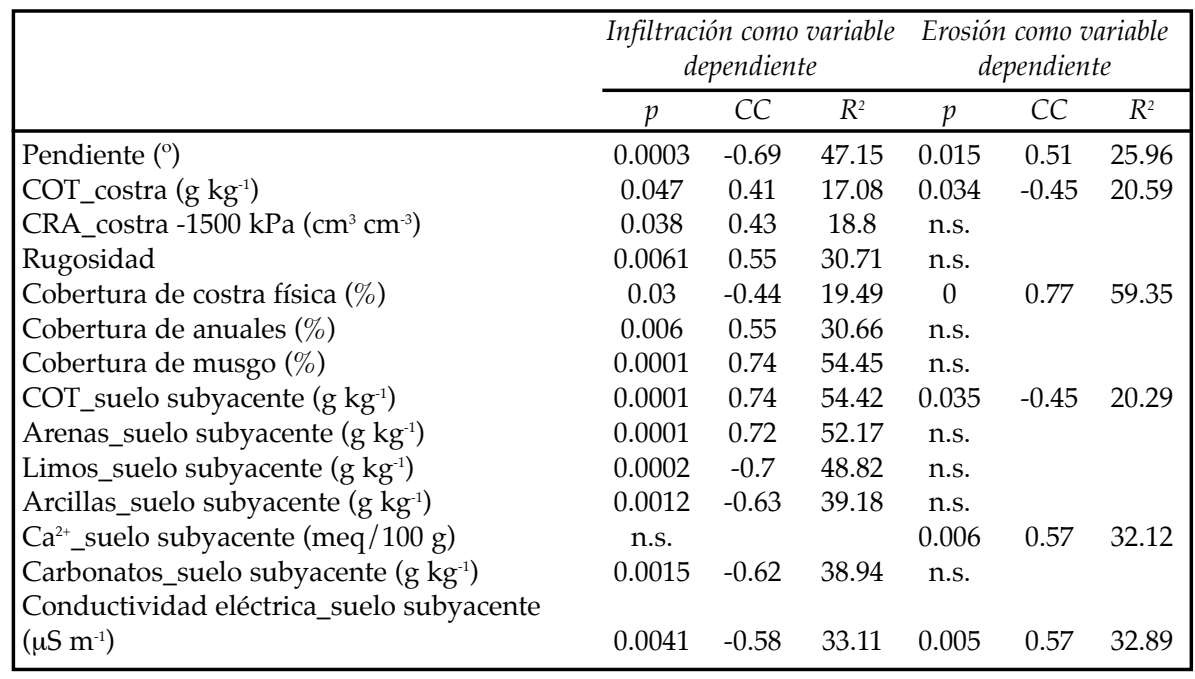


Tabla 5. Variables significativas tras el análisis de regresión simple de las características de la costra y el material subyacente con las tasas de infiltración y erosión, considerando sólo El Cautivo. p: p-valor; CC: coeficiente de correlación; ns: no significativo cuando p es mayor de 0.05. Table 5. Significant variables after conducting a simple regression model of the characteristics of the crust and the underlying material with infiltration and erosion rates, when only El Cautivo is considered. $p$ : $p$ value; CC: correlation coefficient; ns: non-significant when $p$ is higher than 0.05 .

\begin{tabular}{|lcccccc|}
\hline & \multicolumn{3}{c}{$\begin{array}{c}\text { Infiltración como variable } \\
\text { dependiente }\end{array}$} & $\begin{array}{c}\text { Erosión como variable } \\
\text { dependiente }\end{array}$ \\
\cline { 2 - 8 } & $p$ & $C C$ & $R^{2}$ & $p$ & $C C$ & $R^{2}$ \\
\hline Pendiente $\left(^{\circ}\right)$ & 0.0076 & -0.66 & 43.36 & n.s. & & \\
Rugosidad & 0.05 & 0.59 & 34.1 & n.s. & & \\
Cobertura de costra física $(\%)$ & 0.006 & 0.67 & 44.94 & 0.0017 & 0.74 & 54.43 \\
Conductividad eléctrica_suelo subyacente & & & & & & \\
$\left(\mu S \mathrm{~m}^{-1}\right)$ & 0.017 & -0.6 & 36.24 & n.s. & & \\
CIC & n.s. & & & 0.043 & 0.53 & 27.88 \\
CRA -22 kPa_suelo subyacente $\left(\mathrm{cm}^{3} \mathrm{~cm}^{-3}\right)$ & n.s. & & & 0.016 & -0.61 & 36.9 \\
\hline
\end{tabular}

Al realizar el análisis sólo para El Cautivo (Tabla 5), el número de variables significativas obtenidas con los análisis de regresión simple para la infiltración como variable dependiente se reduce a pendiente, rugosidad, cobertura de costra física y conductividad eléctrica del suelo subyacente. Sin embargo, si nos fijamos en la erosión sólo la cobertura de costra física y algunos parámetros del material subyacente mantienen cierto grado de significación.

El análisis de regresión múltiple paso a paso realizado para los dos ecosistemas muestra que el mejor ajuste para la infiltración engloba la cobertura de la costra física, la rugosidad, la pendiente y dos características del material subyacente (el COT y el contenido de arcillas) como variables significativas, con una $\mathrm{r}^{2}$ de 0.67 mientras que para el caso de la erosión, las variables involucradas son la cobertura de la costra física y dos parámetros del material subyacente (el COT y la conductividad eléctrica), con una $\mathrm{r}^{2} \mathrm{de}$ 0.49 .

En el análisis que considera solo El Cautivo, las variables que ajustaron el mejor modelo de regresión para el cálculo de la infiltración fueron la pendiente, la fracción cubierta por costra física y la rugosidad de la costra, con una $\mathrm{r}^{2}$ de 0.62 mientras que para la erosión la $\mathrm{r}^{2}$ fue menor $(0.48)$, y el modelo incluye la fracción de suelo cubierta por costra física y capacidad de intercambio catiónico del material subyacente. 


\section{Discusión}

\subsection{Material subyacente}

Las diferentes características del material subyacente, así como las diferencias topográficas, explican las contrastadas tasas de infiltración y erosión en ambas áreas. Amoladeras es un área llana y con suelos predominantemente arenosos, mientras que El Cautivo es un área de badlands, con una topografía muy irregular y suelos poco desarrollados con un alto contenido en limos y bajo contenido en carbono orgánico, que en su conjunto reducen de forma significativa la porosidad total (MIRALLES et al., enviado), explicando las elevadas tasas de erosión y las bajas tasas de infiltración en estos suelos (Tabla 1), las cuales concuerdan con las ya obtenidas por diferentes autores (SOLÉ-BENET et al., 1997; CANTÓN et al., 2001 \& 2002) en el mismo sitio a diferentes escalas. Las diferencias texturales entre ambas áreas explican también la mayor capacidad de retención de agua en los suelos del Cautivo que en los de Amoladeras. En cuanto a la relación de cada uno de los factores estudiados con la infiltración y la erosión (Tabla 1) resalta la ya conocida influencia de la pendiente en estos procesos (CANTÓN et al., 2001 \& 2002; LÁZARO et al., 2008), con independencia del tipo de costra. El análisis de regresión múltiple por pasos muestra que la pendiente se comporta como una variable predictiva de la infiltración y de la erosión tanto de la costra como del suelo subyacente. Otras características del material subyacente con valor predictivo para la infiltración y la erosión fueron el COT, el contenido en limo (o en arcilla, en el caso de la erosión), el contenido en carbonatos y la conductividad eléctrica. Es conocido el papel que tiene el contenido en carbono orgánico y carbonatos y la textura del suelo en la formación de los agregados del suelo y numerosos autores (MARTÍNEZ-MENA et al., 1998; KIRKBY et al., 2002; MIRALLES et al., 2009) han encontrado correlación de éstos con variables hidrológicas en ecosistemas semiáridos. La conductividad eléctrica aparece como una variable significativa para predecir la infiltración y la erosión, lo cual se explica por las diferencias en esta variable en los dos ecosistemas: la infiltración es mayor y la erosión es menor en Amoladeras, donde el suelo presenta un menor contenido en sales, mientras que en el Cautivo, donde el material parental está formado por margas yesíferas, la conductividad eléctrica del suelo es mucho más elevada, siendo la infiltración menor y la erosión mayor. Dentro de esta área, CANTÓN et al. (2003) encontraron que a medida que aumenta el desarrollo del suelo, la conductividad decrece y también lo hacen la escorrentía y la erosión. El porcentaje de sodio 
intercambiable (PSI), aunque significativo, muestra una relación con la infiltración y la erosión contraria a lo esperado, lo cual puede deberse a la influencia de la proximidad del mar en la zona de Amoladeras. No obstante, el PSI presenta un valor bajo e inferior a $15 \%$, valor a partir del cual puede decirse que el suelo presenta un mayor riesgo de dispersión de arcillas y encostramiento, con los consiguientes problemas en la reducción de la porosidad y del agua infiltrada.

En general, las relaciones estadísticas encontradas entre las propiedades del material subyacente y la infiltración y la erosión después de eliminar la costra son menores de lo esperado. Sin la costra, los primeros milímetros de lluvia, debido al impacto de sus gotas, inducen la formación de un nuevo sellado en la superficie del suelo. La formación de este sello, que tras secarse da lugar a la costra, depende en gran medida del carbono orgánico del suelo, el cual muestra una gran variación dentro de un ecosistema según el tipo de costra y entre ambos ecosistemas. LADO et al. (2004) encontraron menores tasas de infiltración en suelos con menores contenidos en carbono orgánico debido a la menor estabilidad de los agregados, que se rompen y dispersan más fácilmente. La reorganización de las nuevas partículas dispersadas difería en función del contenido en carbono orgánico, resultando una costra más gruesa y compacta en los suelos con bajo contenido. Además, estos autores demostraron la existencia de interacción entre el contenido en $\mathrm{MO}$ y el tamaño de los agregados en la formación de sellados y en las tasas de infiltración.

\subsection{Influencia de las características de la costra}

\subsubsection{Cobertura de la costra biológica y composición}

Las tasas de infiltración son más bajas en las CFSs que en las CBSs. Se sabe que las costras físicas reducen la infiltración y aumentan la escorrentía como consecuencia de una disminución de la estabilidad de los agregados y el cierre de los poros de la matriz del suelo (ROMKENS et al., 1990; ELDRIDGE, 2003), además de la menor rugosidad. Cuando se dan condiciones adecuadas en cuanto a clima, estabilidad de los suelos, etc. estas costras físicas son colonizadas por cianobacterias pioneras y, a medida que la sucesión avanza, entran otras especies de cianobacterias, algas, hongos y finalmente líquenes y musgos. A medida que aumenta la biodiversidad de organismos macroscópicos en la costra, aumenta la rugosidad y, de esta forma, la infiltración. Las costras más evolucionadas (líquenes y musgos) aportan 
mayor C y N a los suelos que las costras de cianobacterias (BARGER, 2003; BELNAP et al., 2008), contribuyendo así a la formación de agregados más estables y a un aumento de la rugosidad de la costra que, en su conjunto, favorecen una mayor infiltración (BELNAP et al., 2005; BELNAP, 2006). Según nuestros resultados, el grado de desarrollo de la costra influye sobre la infiltración y la erosión (Figura 3), con mayores tasas de infiltración y menores tasas de erosión en las costras más desarrolladas (CHAMIZO et al., 2008).

Diversos estudios realizados en diferentes partes del mundo demuestran que las CBSs reducen la erosión (GREENE et al., 1990; ELDRIDGE \& GREENE, 1994; ELDRIDGE \& KINNELL, 1997; ELDRIDGE, 1998; KNAPEN et al., 2007; LÁZARO et al., 2008). Los componentes polisacáridos de los filamentos de las cianobacterias y los geles segregados por los organismos de la costra biológica unen las partículas del suelo, mejorando así la estabilidad del suelo y protegiéndolo de la erosión por el impacto de las gotas de lluvia y la escorrentía (GREENE et al., 1990). Esto concuerda con nuestros resultados, donde también encontramos unas tasas de erosión mucho más elevadas en la costra física y una notable disminución de la producción de sedimentos a medida que aumenta la cobertura de cianobacterias (Figura 2) y que la costra es colonizada por líquenes y musgo (Figura 3). La erosión por el impacto de las gotas de lluvia disgrega las partículas de la superficie del suelo y puede romper los agregados (BARGER et al., 2006). Debido a que las cianobacterias residen justo debajo de la superficie del suelo, éstas son menos efectivas en la protección del suelo frente a la erosión por salpicadura o por los flujos superficiales que los líquenes y musgos, cuyos tejidos cubren la superficie del suelo (TCHOUPOPNOU, 1989; BELNAP, 2006). Dentro de las cianobacterias, las especies más tardías residen justo en la superficie del suelo y ofrecen mayor protección frente a la erosión que las especies de cianobacterias tempranas en la sucesión, como Microcoleus vaginatus, las cuales se encuentran bajo la superficie (GARCIA-PICHEL \& BELNAP, 2001).

Diversos trabajos han demostrado el declive en la erosión por salpicadura a medida que aumenta la cobertura de la costra biótica (ELDRIDGE \& GREENE, 1994) o cuando se comparan suelos encostrados biológicamente con superficies de suelo desnudo (PÉREZ, 1997). En relación a la erosión por escorrentía, ELDRIDGE \& KINNELL (1997) encontraron tasas de erosión hasta cinco veces menores en suelos con costras biológicas que en suelos encostrados físicamente. LÁZARO et al. (2008) también observaron en El Cautivo que la costra dominada por cianobacterias tenía mayor susceptibilidad a la erosión que la costra dominada por líquenes pero que, sin embargo, tras 13 años de seguimiento en esta área, las tasas de erosión sólo fueron significativas en las costras físicas. CANTÓN et al. (2001) encontraron 
en esta misma área, tras un seguimiento de tres años bajo condiciones de lluvia natural, una tasa media de erosión de $21.4 \mathrm{~g} \mathrm{~m}^{-2}$ en superficies cubiertas por líquenes y de $308.1 \mathrm{~g} \mathrm{~m}^{-2}$ en superficies cubiertas por costra física.

Por tanto, podemos destacar el papel importante que tienen las CBSs en la protección del suelo frente a la erosión a escala local.

\subsubsection{Resistencia mecánica de la costra}

Los valores de resistencia medidos en la costra estructural (física) están en el rango de los encontrados por BOUMA \& IMESON (2000) en la costra estructural del regolito margoso en los badlands de Petrer (Alicante) que oscilaron según el tipo de marga entre 0 y $2.25 \mathrm{~kg} \cdot \mathrm{cm}^{-2}$. Dentro de las costras biológicas y a medida que aumenta su desarrollo (en El Cautivo la secuencia de menor a mayor desarrollo sería CI-C-L) disminuye la resistencia de la costra. Estos resultados contrastan con los obtenidos por THOMAS \& DOUGILL (2006) que encontraron que las costras más evolucionadas (con mayor contenido en clorofila y concentración total de N) presentaban mayores resistencias a la penetración. MAESTRE et al. (2002) encontraron mayor resistencia a la penetración a medida que aumentaba la cobertura de cianobacterias, mientras que para hongos y líquenes no era significativa esa relación. En la costra incipiente de cianobacterias se midieron las mayores resistencias. Esto es debido a que esta costra se localiza sobre depósitos de limo en zonas de piedemonte, que suelen tener hasta varios centímetros de grosor y que suelen estar sometidos al pisoteo o al paso de vehículos. No obstante, el método utilizado presenta cierto sesgo: al medir la resistencia de la costra con un penetrómetro, no sólo se mide la resistencia de la propia costra, sino también la resistencia del suelo subyacente. En las CBSs más desarrolladas es posible que el penetrómetro sólo afecte a la costra pero en las más incipientes casi toda la resistencia a la penetración proviene de fracción mineral compactada de la superficie del suelo. Esta limitación debe tenerse en cuenta cuando se emplean parámetros de resistencia a la hora de modelizar la escorrentía y la erosión en áreas encostradas.

\subsubsection{Rugosidad e hidrofobia de la costra}

Las CBSs influyen sobre la microtopografía del suelo, aumentando la rugosidad y actuando como estructuras de retención de agua que pueden por tanto favorecer la infiltración. En nuestro caso, la rugosidad aumenta a 
medida que aumenta la evolución de la costra biológica. KIDRON (2007) también encontró un aumento de la rugosidad con el desarrollo de la costra y una mayor biomasa de ésta. La infiltración también aumenta con el desarrollo de la costra, excepto en la costra de líquenes en El Cautivo. La erosión también disminuye claramente a medida que la costra es más evolucionada. Los líquenes promueven una mayor rugosidad de la superficie que las cianobacterias y, por tanto, pueden aumentar más la infiltración. Sin embargo, nuestros resultados muestran que la escorrentía comienza antes y es mayor en la costra de líquenes, coincidiendo con resultados previos en el mismo área (ALEXANDER \& CALVO-CASES, 1990; SOLÉ-BENET et al., 1997; CANTÓN et al., 2001 \& 2002) que apuntan que una extensa superficie del suelo cubierta por líquenes crustáceos y escuamolosos reducen la infiltración. Esto puede deberse a diversos factores, como la pendiente y la mayor hidrofobia de esta costra. En el Cautivo la distribución espacial de los tipos de cubiertas del suelo está fuertemente controlada por la topografía (LÁZARO et al., 2000; CANTÓN et al., 2004). Los líquenes cubren laderas con pendientes muy elevadas, sólo ligeramente inferiores a las de las laderas erosionadas con costra estructural, mientras que las cianobacterias y la costra deposicional con cianobacterias incipientes aparecen en piedemontes y geoformas con pendientes más suaves. Otra razón puede ser el comportamiento hidrofóbico de la costra liquénica, pese a que en los análisis no aparece como una variable predictora importante para la infiltración. Las CBSs pueden reducir la infiltración por diversos mecanismos: 1) el cierre de los poros de la superficie del suelo, 2) el hinchamiento de las cianobacterias en la costra cuando se mojan (ELDRIDGE, 2003), y 3) propiedades hidrofóbicas inherentes de algunas costras (YAIR, 2003). Los organismos de la costra biológica se expanden cuando se mojan, provocando el cierre de los poros de la matriz del suelo y originando superficies hidrofóbicas (ROBERTS \& CARBON, 1972; KIDRON et al., 1999). KIDRON et al. (1999) encontraron que la escorrentía se generaba rápidamente en las CBSs debido a la hidrofobia de las algas. DE PLOEY (1980) observó, en dunas arenosas en Kalmthout (Bélgica), una disminución en las tasas de infiltración debido a la colonización por algas y hongos y la hidrofobia inducida por los mismos. En El Cautivo, a medida que aumenta la biomasa de líquenes formando una costra continua en la superficie del suelo, mayor es el agua retenida entre las hifas del líquen y la superficie de los talos, pudiendo reducir así la infiltración y favorecer los procesos de escorrentía (SOUZA-EGIPSY et al., 2002). Las cianobacterias, en cambio, consisten en filamentos en la superficie del suelo, dejando espacios libres entre ellos, y por tanto son menos probables que ocupen los poros del suelo a diferencia de líquenes y musgos (BELNAP, 2006). 


\subsection{Relación de las características de la costra con la infiltración y la erosión}

Tanto si consideramos las dos áreas o sólo El Cautivo, las características de la costra con mayor valor predictivo para la infiltración son la cobertura de costra física y la rugosidad, mientras que para la erosión es la cobertura de costra física. Además, otras características relacionadas con el material subyacente, como el contenido en carbono orgánico y en arcilla cuando consideramos los dos ecosistemas, o la conductividad eléctrica cuando sólo se considera El Cautivo, se muestran como variables significativas. En todos los casos, la pendiente tiene un alto valor predictivo para la infiltración y la erosión. En general, la varianza absorbida en las relaciones estadísticas entre la infiltración y las características de la costra y entre éstas y la erosión es baja, lo que puede deberse a las complejas interacciones entre factores. Por ejemplo, la cobertura de líquenes presenta una respuesta diferente sobre la infiltración en los dos ecosistemas a pesar de que en ambos abundan las mismas especies; eso puede deberse a que: a) en El Cautivo los líquenes aparecen en pendientes más elevadas y presentan una cobertura continua de éstos, por lo que actúan disminuyendo la infiltración, pese a tratarse de la costra más evolucionada y con una mayor rugosidad; b) en Amoladeras, la topografía es llana y junto al hecho de que los líquenes suelen estar rotos debido al pisoteo del ganado, muy frecuente en esta zona, hacen que la infiltración sea mayor en esta costra si la comparamos con la costra dominada por cianobacterias, más resistente al pisoteo. La interacción de todos estos factores, junto con las limitaciones de los análisis estadísticos aplicados y los métodos de campo usados pueden explicar el limitado valor predictivo de las características analizadas. Sólo se han usado regresiones lineales para el análisis de estas relaciones y en algunos casos, la aplicación de modelos no lineales puede ser más realista. Probablemente, el uso de modelos múltiples no lineales podría proveer mejores resultados, sin embargo, es difícil aplicar estos análisis cuando se dispone de un número no muy elevado de datos o las relaciones entre factores es compleja.

\section{Conclusiones}

Las complejas interacciones entre factores, por un lado relacionados con la costra, y por otro lado, con el material subyacente, hacen difícil establecer los factores que determinan la respuesta hidrológica y erosiva de las costras biológicas frente a la infiltración y la erosión. No obstante, existen diferentes variables significativamente relacionadas con estos procesos. 
La pendiente, junto con la litología, tiene un alto valor de predicción para la infiltración y la erosión de superficies encostradas. La infiltración es mayor y la erosión es menor en Amoladeras, donde la topografía es llana y los suelos son arenosos, que en El Cautivo, un área de badlands con elevadas pendientes y suelos con un alto contenido en limo. La pendiente aparece como uno de los factores que puede explicar las bajas tasas de infiltración en la costra de líquenes en El Cautivo a pesar de su mayor contenido en materia orgánica y su mayor rugosidad. Respecto a las características del material subyacente, los modelos de regresión linear múltiple identifican el contenido en carbono orgánico como la variable que más significativamente influye sobre la infiltración y la erosión. Respecto a las características de la costra, la cobertura de costra física y la rugosidad son las variables más predictivas, aumentando la infiltración a medida que la cobertura de costra física disminuye y la rugosidad aumenta. En el caso de la erosión, la cobertura de costra física es la variable más determinante, disminuyendo la erosión a medida que la cobertura de costra física disminuye y, por ende, la cobertura de costra biótica aumenta.

En general, aplicando modelos de regresión lineales, la varianza absorbida en la predicción es reducida debido a la compleja interacción entre factores y posiblemente, debido a la existencia de relaciones no lineales entre variables. Para este propósito, la aplicación de modelos no lineales podría mejorar los resultados, lo que constituye el siguiente paso a este trabajo. Además, estos resultados corresponden a eventos de precipitación extremos, de manera que en condiciones de lluvia natural los continuos cambios de intensidad y de humedad antecedente pueden afectar a las interacciones descritas y modificar las variables que aparecen como más predictivas, por lo que se hace imprescindible analizar la influencia de las costras y características asociadas en condiciones de lluvia natural.

\section{Agradecimientos}

Este trabajo ha recibido el apoyo financiero de diferentes proyectos de investigación: PROBASE (Ref.: CGL2006-11619/HID), PREVEA (Ref.: CGL2007-63258/BOS), financiados por el Plan Nacional de I+D y COSTRAS (Ref. RNM 3614), financiado por la Junta de Andalucía y EC-DG RTD- 6th Framework Research Programme (1.1.6.3)-Research on Desertificationproject DESIRE (037046). 


\section{Referencias}

ALEXANDER, R.W. \& CALVO-CASES, A., 1990. The influence of lichens on slope processes in some Spanish badlands. In: THORNES, J.B. (Ed.): Vegetation and erosion: Process and Environments, Wiley, Chichester, UK, 385398.

AUZET, A.V., KIRKBY, M.J. \& VAN DIJK, P., 2005. Surface characterisation for soil erosion forecasting. Catena, 62 (2): 77-78.

BARAHONA, E., FERNÁNDEZ, J. \& MINGORANCE, M.D., 2005. Determinación rápida de carbono orgánico en suelos por oxidación vía húmeda. Control de degradación de suelos, coord. por Raimundo Jiménez Ballesta, Ana María Alvárez González, Vol. 1, (Libro de actas), ISBN 84-689-2620-5, 737741.

BARGER, N.N., 2003. Biogeochemical cycling and $N$ dynamics of biological soil crusts in a semi-arid ecosystem. Ph.D. thesis. Colorado State University, Fort Collins, CO.

BARGER, N. N., HERRICK, J. E., VAN ZEE, J. \& BELNAP, J., 2006. Impacts of biological soil crust disturbance and composition on $\mathrm{C}$ and $\mathrm{N}$ loss from water erosion. Biogeochemistry, 77 (2): 247-263.

BELNAP, J., 2006. The potential roles of biological soil crusts in dryland hydrologic cycles. Hydrological Processes, 20 (15): 3159-3178.

BELNAP , J., BUDEL, B. \& LANGE, O.L., 2001. Biological soil crusts: Characteristics and distribution. In: BELNAP, J. \& LANGE, O.L. (Eds.): Biological Soil Crusts: Structure, Function and Management, Springer-Verlag, Berlin, 3-30.

BELNAP, J., PHILLIPS, S. L., WITWICKI, D. L. \& MILLER, M. E., 2008. Visually assessing the level of development and soil surface stability of cyanobacterially dominated biological soil crusts. Journal of Arid Environments, 72 (7): 1257-1264.

BELNAP, J., WELTER, J.R., GRIMM, N.B., BARGER, N. \& LUDWIG, J.A., 2005. Linkages between microbial and hydrologic processes in arid and semiarid watersheds. Ecology, 86 (2): 298-307.

BOUMA, N. A. \& IMESON, A. C., 2000. Investigation of relationships between measured field indicators and erosion processes on badland surfaces at Petrer, Spain. Catena, 40: 147-171.

CALVO-CASES, A., GISBERT, B., PALAU, E. \& ROMERO, M., 1988. Un simulador de lluvia de fácil construcción. In: Sala, M. \& Gallart, F. (Eds): Métodos y técnicas para la medición en el campo de procesos geomorfológicos, Vol 1, Sociedad Española de Geomorfología, Zaragoza, 6-15.

CANTÓN, Y., DEL BARRIO, G., SOLE-BENET, A. \& LÁZARO, R., 2004. Topographic controls on the spatial distribution of ground cover in a semiarid badlands area. Catena, 55: 341-365. 
CANTÓN, Y., DOMINGO, F., SOLÉ-BENET, A. \& PUIGDEFÁBREGAS, J., 2001. Hydrological and erosion response of a badlands system in semiarid SE Spain. Journal of Hydrology, 252 (1-4): 65-84.

CANTÓN, Y., DOMINGO, F., SOLÉ-BENET, A. \& PUIGDEFÁBREGAS, J., 2002. Influence of soil surface types on the overall runoff of the Tabernas badlands (SE Spain). Field data and model approaches. Hydrological Processes, 16: 2621-2643.

CANTÓN, Y., SOLÉ-BENET, A. \& LÁZARO, R., 2003. Soil-geomorphology relations in gypsiferous materials of the Tabernas desert (Almería, SE Spain). Geoderma, 115(3-4): 193-222.

CHAMIZO, S., CANTÓN, Y., LÁZARO, R., SOLÉ-BENET, A. \& DOMINGO, F., 2008. Soil crusting effects on infiltration under extreme rainfall in semiarid environments. XII Biennal International Conference, Hydrological extremes in small basins, 18-20 September, Cracow, Poland.

DEBANO, L.F., 1981. Water repellent soils: a state of art. Gen. Tech. Rpt. PSW-46. Berkeley, CA: U.S. Department of Agriculture, Forest Service, Pacific Southwest Forest and Range Experiment Station, 21 pp.

DE PLOEY, J., 1980. Some field measurements and experimental data on wind-blown sands. In: BOODT, M. \& GABRIELS, D. (Eds): Assessment of Erosion, Wiley, New York, 541-552.

ELDRIDGE, D.J., 1998. Trampling of microphytic crusts on calcareous soils, and its impact on erosion under rain-impacted flow. Catena, 33: 221-239.

ELDRIDGE, D.J., 2003. Biological soil crusts and water relations in Australian deserts. In: BELNAP, J. \& LANGE, O.L. (Eds.): Biological Soil Crusts: Structure, Function, and Management, Springer-Verlag, Berlin, 315-325.

ELDRIDGE, D.J. \& GREENE, R.S.B., 1994. Assessment of sediment yield by splash erosion on a semi-arid soil with varying cryptogam cover. Journal of Arid Environments, 26 (3): 221-232.

ELDRIDGE, D.J. \& KINNELL, P.I.A, 1997. Assessment of erosion rates from microphyte dominated calcareous soils under rain-impacted flow. Australian Journal of Soil Research, 35 (3): 475-489.

ELDRIDGE, D.J., ZAADY, E. \& SHACHAK, M., 2000. Infiltration through three contrasting biological soil crusts in patterned landscapes in the Negev, Israel. Catena, 40: 323-336.

FOX, D.M., LE BISSIONAIS, Y. \& QUÉNTIN, P., 1998. The implications of spatial variability in surface seal hydraulic resistance for infiltration in a mound and depression microtopography. Catena, 32 (2): 101-114.

GARCIA-PICHEL, F. \& BELNAP, J., 2001. Small scale environments and distribution of biological soil crusts. In: BELNAP, J. \& LANGE, O.L. (Eds): Biological Soil Crusts: Structure, Function, and Management, Springer-Verlag, Berlin, Heidelberg, 193-202. 
GEE, G.W. \& BAUDER, J.W., 1986. Particle-size analysis. In: American Society of Agronomy-Soil Science Society of America. Methods of soil analysis, part.1. Physical and mineralogical methods- Agronomy monograph, 9 (2nd. edition): 383-411.

GREENE R.S.B., CHARTRES, C.J. \& HODGKINSON, K.C., 1990. The effects of fire on the soil in a degraded semiarid woodland. I. Cryptogam cover and physical and micromorphological properties. Australian Journal of Soil Research, 28: 755-777.

KIDRON, G.J., 2007. Millimeter-scale microrelief affecting runoff yield over microbiotic crust in the Negev Desert. Catena, 70: 266-273.

KIDRON, G.J., YAALON, D.H. \& VONSHAK, A., 1999. Two causes for runoff initiation on microbiotic crusts: hydrophobicity and pore clogging. Soil Science, 164: 18-27.

KIDRON, G.J. \& YAIR, A., 2001. Runoff-induced sediment yield over dune slopes in the Negev Desert. 1: Quantity and variability. Earth Surface Processes and Landforms, 26: 461-474.

KIRKBY, M., BRACKEN, L. \& REANEY, S., 2002. The influence of land use, soils and topography on the delivery of hillslope runoff to channels in SE Spain. Earth Surface Processes and Landforms, 27: 1459-1473.

KLEVERLAAN, K., 1989. Neogene history of the Tabernas basin (SE Spain) and its Tortonian submarine fan development. Geologie en Mijnbouw, 68: 421-432.

KNAPEN, A., POESEN, J., GALINDO-MORALES, P., DE BAETS, S. \& PALS, A., 2007. Effects of microbiotic crusts under cropland in temperate environments on soil erodibility during concentrated flow. Earth Surface Processes and Landforms, 32: 1884-1901.

LADO, M., PAZ, A. \& BEN-HUR, M., 2004. Organic matter and aggregate size interactions in infiltration, seal formation, and soil loss. Soil Science Society of America Journal, 68: 935-942.

LARK, R.M., BISHOP, T.F.A. \& WEBSTER, R., 2007. Using expert knowledge with control of false discovery rate to select regressors for prediction of soil properties. Geoderma, 138 (1-2): 65-78.

LÁZARO, R., ALEXANDER, R.W. \& PUIGDEFABREGAS, J., 2000. Cover distribution patterns of lichens, annuals and shrubs in the Tabernas Desert, Almería, España. In: ALEXANDER, R.W. \& MILLINGTON, A.C. (Eds.): Vegetation Mapping: From Patch to Planet, Wiley, Chichester, 19-39.

LÁZARO, R., CANTÓN, Y., SOLÉ-BENET, A., BEVAN, J., ALEXANDER, R., SANCHO, L.G. \& PUIGDEFABREGAS, J., 2008. The influence of competition between lichen colonization and erosion on the evolution of soil surfaces in the Tabernas badlands (SE Spain) and its landscape effects. Geomorphology, 102: 252-266. 
LÁZARO, R. \& REY, J.M., 1990. Sobre el clima de la provincia de Almería (SE Ibérico): primer ensayo de cartografía automática de medias anuales de temperatura y precipitación. Suelo y Planta, 61-68.

LÁZARO, R., RODRIGO, F.S., GUTIÉRREZ, L., DOMINGO, F. \& PUIGDEFÁBREGAS, J., 2001. Analysis of a 30-year rainfall record (1967-1997) in semi-arid SE Spain for implications on vegetation. Journal of Arid Environments, 48: 373-395.

LE BISSIONAIS, Y., CERDAN, O., LECOMTE, V., BENKHADRA, H., SOURCHÉRE, V. \& MARTIN, P., 2005. Variability of soil surface characteristics influencing runoff and interrill erosion. Catena, 62 (2-3): 111124.

LOEPPERT, R.H. \& SUAREZ, D.L., 1996. Carbonate and gypsum. In: SPARKS, D.L. (Ed.): Methods of Soil Analysis. Part 3, Chemical Methods, SSSA-ASA, Madison, WI, 437-474.

MAESTRE, F.T., HUESCA, M., ZAADY, E., BAUTISTA, S. \& CORTINA, J., 2002. Infiltration, penetration resistance and microphytic crust composition in contrasted microsites within a Mediterranean semi-arid steppe. Soil Biology \& Biochemistry, 34: 895-898.

MARTÍNEZ-MENA, M., WILLIAMS, A. G., TERNAN, J. L. \& FITZJOHN, C., 1998. Role of antecedent soil water content on aggregates stability in a semi-arid environment. Soil \& Tillage Research, 48: 71-80.

MIRALLES, I., ORTEGA, R., ALMENDROS, G., SÁNCHEZ-MARAÑÓN, M. \& SORIANO, M., 2009. Soil quality and organic carbon ratios in mountain agroecosystems of South-east Spain. Geoderma, 150: 120-128.

MIRALLES, I., CANTÓN, Y. \& SOLÉ-BENET, A. (submitted to SSSAJ). Macroporosity of crusted soils as an indicator of soil quality. Application in a semiarid badlands ecosystem.

NDIAYE, B., ESTEVES, M., VANDERVAERE, J.P., LAPETITE, J.M. \& VAUCLIM, M., 2005. Effect of rainfall and tillage direction on the evolution of surface crusts, soil hydraulic properties and runoff generation for a sandy loam soil. Journal of Hydrology, 307 (1-4): 294-311.

RICHARDS, L.A., 1945. Pressure-membrana apparatus and use. Agricultural Engineering, 28: 451-454.

ROBERTS, F.J. \& CARBON, B.A., 1972. Water repellence in sandy soils of south-western Australia. Australian Journal of Soil Research, 10: 35-42.

ROMKENS, M.J.M., PRASSAD, S.N. \& WHISLE, F.D., 1990. Surface sealing and infiltration. In: ANDERSON, M.G. \& BURT, I.P. (Eds): Process studies in hillslope hydrology, Wiley, New York, 127-172.

SOlÉ-BENET, A., CALVO, A., CERDÁ, A., LÁZARO, R., PINI, R., \& BARBERO, J., 1997. Influences of micro-relief patterns and plant cover on 
runoff related processes in badlands from Tabernas (SE Spain). Catena, 31(1-2): 23-38.

SOUZA-EGIPSY, V., ASCASO, C. \& SANCHO, L.G., 2002. Water distribution within terricolous lichens revealed by scanning electron microscopy and its relevance in soil crust ecology. Mycological Research, 106 (11): 1367-1374.

SUMNER, M.E., \& MILLER, W.P., 1996. Cation exchange capacity and exchange coefficients. In: SPARKS, D.L. (Ed.): Methods of Soil Analysis. Part , Chemical Methods, SSSA-ASA, Madison. WI: 1201-1229.

TCHOUPOPNOU, E., 1989. Experimental studies of rainsplash erosion from soil mycrophytic crusts on Utah rangelands. M.Sc. thesis, Utah, State University.

THOMAS, A.D. \& DOUGILL, A.J., 2006. Distribution and characteristics of cyanobacterial soil crusts in the Molopo Basin, South Africa. Journal of Arid Environments, 64: 270-283.

VAN SCHAIK, N.L.M.B., 2009. Spatial variability of infiltration patterns related to site characteristics in a semi-arid watershed. Catena, 78: 36-47.

VESTE, M., 2005. The importance of biological soil crusts for rehabilitation of degraded arid and semi-arid ecosystems. Science of Soil and Water Conservation, 3: 42-47.

WARREN, S.D., 2003. Synopsis: influence of biological soil crusts on arid land hydrology and soil stability. In: BELNAP, J. \& LANGE, O.L. (Eds.): Biological Soil Crusts: Structure, Function, and Management, Springer-Verlag, Berlin, 349-360.

WALKLEY, A.J. \& BLACK, I.A., 1934. Estimation of soil organic carbon by the chromic acid titration method. Soil Science, 37, 29.

YAIR, A., 2003. Effects of biological soil crusts on water redistribution in the Negev desert, Israel: a case study in longitudinal dunes. In: BELNAP, J. \& LANGE, O.L. (Eds.): Biological Soil Crusts: Structure, Function, and Management, Springer-Verlag, Berlin, 303-314.

YAIR, A., 2008. Effects of surface runoff and subsurface flow on the spatial variability of water resources in longitudinal dunes. In: VESTE, M. (Ed.): Arid Dune Ecosystems-the Nizzana Sands in the Negev Desert, Ecol. Stud., 200. Springer, Berlin, 251-269. 